\section{Self-sampling and self-testing for STls and HIV: the case for consistent nomenclature}

\author{
Emma M Harding-Esch, ${ }^{1,2}$ Emma Hollis, ${ }^{1}$ Hamish Mohammed, ${ }^{1}$ \\ John M Saunders ${ }^{1}$
}

At the recent BASHH Annual Conference, there were numerous presentations referring to home-sampling or testing, oftentimes interchangeably and inconsistently, despite these being distinct methodologies. Furthermore, by specifying 'home' as opposed to 'self', the sampling or testing is constrained to the home environment. With diagnostic advances enabling modified care pathways, we urge that these terms be used consistently.

The WHO defines HIV self-testing as 'a process whereby a person who wants to know his or her HIV status collects a specimen, performs a test and interprets the test result in private'. ${ }^{1}$ In contrast, selfsampling requires individuals to send their specimens to a laboratory where they are

${ }^{1}$ HIVISTI Department, National Infection Service, Public Health England, London, UK; ${ }^{2}$ Applied Diagnostic Research and Evaluation Unit, Institute for Infection and Immunity, St George's University of London, London, UK

Correspondence to Dr Emma M Harding-Esch, HIVI STI Department, National Infection Service, Public Health England, London, UK;

Emma.Harding-Esch@phe.gov.uk tested, and the laboratory returns the test result to the individual.

Self-sampling for HIV and STIs is currently available in the UK from a number of public and private providers. Self-testing is a nascent field, with only one HIV self-test currently licensed in the UK and validated self-tests for STIs still in development (http://www.esti2.org.uk).

Consistent terminology is crucial given the implications for research, policies, regulations, intervention development, programme delivery and care pathway provision (including result confirmation, treatment and partner notification). For surveillance, Public Health England's genitourinary medicine clinic activity dataset (GUMCADv2) surveillance system introduced the REF3 surveillance code in 2015 to identify individuals referred from self-testing and sampling services. While enabling an assessment of reactive test results from self-collected specimens, this code does not differentiate between selfsampling and self-testing, and will therefore need modification as self-testing expands to ensure the collection of robust surveillance data. $^{2}$
As options for STI and HIV selfsampling and self-testing continue to expand, we urge colleagues to be consistent and to define the terminology used in order to limit confusion for both professionals and patients.

Twitter Follow Emma Harding-Esch at @eSTI2_org and John Saunders at @saunders_j

Contributors EMH-E wrote the manuscript, and $\mathrm{EH}$, HM and JMS assisted.

Funding EMH-E is funded by the UK Clinical Research Collaboration (Medical Research Council) Translation Infection Research Initiative Consortium (grant number G0901608).

Competing interests None declared.

Provenance and peer review Not commissioned; internally peer reviewed.

To cite Harding-Esch EM, Hollis E, Mohammed $\mathrm{H}$, et al. Sex Transm Infect Published Online First: [please include Day Month Year] doi:10.1136/sextrans-2016052841

Received 12 August 2016

Accepted 18 August 2016

Sex Transm Infect 2016;0:1

doi:10.1136/sextrans-2016-052841

\section{REFERENCES}

1 WHO. HIV self-testing. Supplementary section to the 2013 WHO consolidated guidelines on the use of antiretroviral drugs for treating and preventing HIV infection, Chapter 5 - HIV diagnosis and ARV drugs for HIV prevention. 2013. http://www.who.int/hiv/pub/guidelines/arv2013/ arv2013supplement to chapter05.pdf

2 Harding-Esch E, Nardone A, Gibbs J, et al. Can remote STI/HIV testing and eClinical Care be compatible with robust public health surveillance? $D H^{\prime} 15$ : proceedings of the 5th International Conference on Digital Health 2015 : May 18-20, 2015, Florence, Italy/in cooperation with: ACM SIGMOD \& ACM KDD International Conference on Digital Health (5th: 2015: Florence, Italy); 2015:129-30. 